\title{
The Impact of Warfarin on Patients with End Stage Renal Disease
}

\author{
Anahita Dua, ${ }^{1}$ Sapan S. Desai, ${ }^{2}$ Harvey J. Woehlck, ${ }^{3}$ and Cheong J. Lee ${ }^{1}$ \\ ${ }^{1}$ Department of Surgery, Medical College of Wisconsin, Milwaukee, WI 53226, USA \\ ${ }^{2}$ Department of Vascular Surgery, Southern Illinois University, Springfield, IL 62901, USA \\ ${ }^{3}$ Department of Anesthesiology, Medical College of Wisconsin, Milwaukee, WI 53226, USA
}

Correspondence should be addressed to Anahita Dua; anahita.dua@uth.tmc.edu

Received 16 July 2014; Revised 17 August 2014; Accepted 28 August 2014; Published 8 September 2014

Academic Editor: Arnon Blum

Copyright (C) 2014 Anahita Dua et al. This is an open access article distributed under the Creative Commons Attribution License, which permits unrestricted use, distribution, and reproduction in any medium, provided the original work is properly cited.

\begin{abstract}
Introduction. A deficiency in vitamin $\mathrm{K}$ through the utilization of warfarin may result in increased vascular calcification and complications. This study aimed to determine the impact of warfarin administration on patients with end stage renal disease (ESRD) in a large, national sample. Methods. A retrospective analysis using the 2005-2010 National Inpatient Sample (NIS), a part of the Health Care Utilization Project (HCUP), was completed using ICD-9 diagnosis codes to capture patients with ESRD prescribed and not prescribed warfarin. Statistical analysis was through ANOVA and chi-squared testing. Results. From 2005-2010, 927,814 patients with ESRD were identified nationally. 3.5\% $(32,737)$ were prescribed warfarin. Patients prescribed warfarin had an average age of 64 years and 51\% were male. For every comorbid condition (amputation, congestive heart failure, chronic obstructive pulmonary disorder, cerebrovascular accident, diabetes, hypertension, myocardial infarction, peripheral vascular diasese, and valvular disease) patients prescribed Warfarin had significantly higher rates of disease as compared to their nonwarfarin ESRD counterparts. ESRD patients prescribed warfarin had significantly shorter length of stay but increased hospital charges. They were more likely to be discharged to home and had significantly decreased in-hospital mortality. Conclusion. Patients with ESRD taking warfarin are more likely to have comorbidities and/or complications but have a decreased LOS and in-hospital mortality compared to their ESRD counterparts not administered warfarin.
\end{abstract}

\section{Introduction}

Vascular calcification is associated with increasing age and advancing atherosclerotic plaque [1]. While the arterial system is impacted systemically by this pathology, calcification of the coronary vasculature specifically has been correlated with an increased risk of cardiac events rooted in plaque instability and subsequent rupture [2]. Patients with end stage renal disease (ESRD) have a 2- to 5-fold increase in coronary artery calcification compared to healthy, agematched individuals, which results in a 10-30 times greater risk of mortality from cardiovascular events [1,2].

While vascular calcification in ESRD patients was originally considered to be a passive process resulting from progressive inflammation and subsequent atherosclerotic development, newer data suggests that active upregulation of various bone matrix proteins and calcium-regulatory hormones is instrumental in the development of vascular calcification [2].

Vitamin $\mathrm{K}$ is an essential factor to a hepatic gammaglutamyl carboxylase that adds a carboxyl group to glutamic acid residues on clotting factors II, VII, IX, and X, protein S, protein $\mathrm{C}$, and protein $\mathrm{Z}$ and is integral to the clotting cascade. Oral anticoagulants inhibit vitamin $\mathrm{K}$ epoxide reductase, thereby preventing vitamin $\mathrm{K}$ from recycling back to its active form resulting in a hypocoaguable state. Aside from the impact on clotting, vitamin $\mathrm{K}$ has also been shown to both impair the physiological effect of osteocalcin, which regulates bone mineralization, and activate the Matrix G1A protein (MGP) which inhibits calcification in vascular tissue [3-6]. A deficiency in active vitamin $\mathrm{K}$ through long-term utilization of warfarin may result in increased vascular calcification [7]. 
This vascular calcification especially in the coronaries may be particularly hazardous in ESRD patients given the already increased risk of cardiovascular events.

This study aimed to determine the impact of warfarin administration on patients with ESRD in a large, national sample.

\section{Methods}

2.1. Database and Selection. A retrospective analysis was completed using the National Inpatient Sample (NIS), a part of the Health Care Utilization Project (HCUP) that is maintained by the Agency for Healthcare Research and Quality (AHRQ) [7]. The NIS is the largest all-payer inpatient database and it includes a stratified $20 \%$ random sample of all nonfederal inpatient hospital admissions throughout the United States. Clinical records between 2005 and 2010 were derived using the ninth revision of the International Classification of Diseases (ICD-9) diagnosis codes to ensure that the sample included patients with end stage renal disease (ESRD).

2.2. Variables. The independent variable was years, ranging from 2005 to 2010. The year 2005 was chosen for the start of the analysis as this is the first year that ESRD is coded separately in the NIS. Demographic covariates included age, gender, and race. Clinical covariates included comorbid conditions such as amputation, congestive heart failure $(\mathrm{CHF})$, chronic obstructive pulmonary disease (COPD), cerebrovascular accident (CVA), diabetes mellitus (DM), hypertension (HTN), myocardial infarction (MI), peripheral vascular disease (PVD), and valvular disorders. Hospital covariates included median length of stay (LOS) in days and median hospital charges adjusted for 2010 US dollars using the consumer price index (CPI). Disposition included discharge to home, discharge to an acute care facility, discharge to a rehabilitation/skilled nursing facility, and in-hospital mortality.

2.3. Statistical Analysis. Statistical analysis was completed using analysis of variance (ANOVA) for continuous variables (i.e., age) and chi-squared for categorical variables (i.e., gender, race, and mortality). The Mann-Whitney $U$ test was used for LOS and total charges. Data analysis and management were completed using the IBM SPSS software package (SPSS version 21.0, SPSS Inc., Chicago, IL, USA). Statistical significance was set at a probability of $P<0.05$. Population estimates were made using discharge sampling weights included within the NIS and data published by the US Census Bureau. Values are presented as mean $+/-$ standard deviation for age, or as median with interquartile range (IQR) in parentheses.

\section{Results}

From the 2005-2010 study period, 927,814 patients with ESRD were identified nationally. Of these, 3.5\% (32,737) were prescribed warfarin. Patients prescribed warfarin had
TABLE 1: Comparison of the demographics and comorbidities of patients with end stage renal disease with and without warfarin from the 2005-2010 National Inpatient Sample.

\begin{tabular}{|c|c|c|c|}
\hline & $\begin{array}{l}\text { ESRD with } \\
\text { warfarin }\end{array}$ & $\begin{array}{l}\text { ESRD without } \\
\text { warfarin }\end{array}$ & $P$ \\
\hline \multirow[t]{2}{*}{ Number } & 32,737 & 895,077 & \\
\hline & \multicolumn{3}{|c|}{ Demographics } \\
\hline Age in years $\pm S D$ & $64.1 \pm 15.7$ & $61.1 \pm 16.1$ & $<0.001$ \\
\hline Female (\%) & $49.0 \%$ & $48.0 \%$ & NS \\
\hline \multirow[t]{2}{*}{ Caucasian (\%) } & $54.6 \%$ & $42.8 \%$ & $<0.001$ \\
\hline & \multicolumn{3}{|c|}{ Comorbid conditions } \\
\hline Amputation & $4.6 \%$ & $3.6 \%$ & $<0.001$ \\
\hline $\mathrm{CHF}$ & $23.0 \%$ & $21.0 \%$ & $<0.001$ \\
\hline COPD & $21.0 \%$ & $18.0 \%$ & $<0.001$ \\
\hline CVA & $7.9 \%$ & $3.8 \%$ & $<0.001$ \\
\hline $\mathrm{DM}$ & $28.0 \%$ & $25.0 \%$ & $<0.001$ \\
\hline HTN & $76.0 \%$ & $75.0 \%$ & $<0.001$ \\
\hline MI & $8.6 \%$ & $6.0 \%$ & $<0.001$ \\
\hline PVD & $16.0 \%$ & $15.0 \%$ & $<0.001$ \\
\hline Valve & $13.0 \%$ & $5.0 \%$ & $<0.001$ \\
\hline
\end{tabular}

an average age of 64 years; $51 \%$ were male and $54.6 \%$ of the cohort were Caucasian.

For every comorbid condition (amputation, CHF, COPD, CVA, DM, HTN, MI, PVD, and valvular disease), patients prescribed warfarin had significantly higher rates of disease compared to their nonwarfarin, ESRD counterparts. Table 1 summarizes these findings.

ESRD patients prescribed warfarin have significantly shorter LOS but increased overall hospital charges. They were more likely to be discharged to home and had significantly decreased in-hospital mortality. These findings are summarized in Table 2.

\section{Discussion}

ESRD patients have relative depletion in vitamin $\mathrm{K}$ and this may explain the accelerated medial calcinosis [1-3]. Utilization of warfarin which works by inhibiting conversion of vitamin $\mathrm{K}$ into the active form may be further detrimental to this patient population.

The incidence of ESRD is increasing in the USA. Between 1980 and 2009, there was a $600 \%$ increase in the rate of ESRD from 290 to 1,738 cases per million. ESRD is a major contributor to morbidity and mortality. Mortality rates related to ESRD have increased from 10,478 in 1980 to 90,118 in 2009. In patients with ESRD, cardiovascular events are the leading cause of death $[2,8]$.

Patients undergoing dialysis are up to 30 times more likely to die from a cardiac event in comparison to the general public; hence, the aggressive strategies are implemented in this patient cohort to mitigate this risk of mortality [2]. There is controversy surrounding the cause of this increased cardiovascular risk [2]. Although there is an increased prevalence of Framingham risk factors in ESRD patients, specific 
TABLE 2: Comparison of the hospital parameters and disposition of patients with end stage renal disease with and without warfarin from the 2005-2010 National Inpatient Sample.

\begin{tabular}{lccc}
\hline \multicolumn{3}{c}{ Hospital parameters } \\
\hline LOS & $4(2-7)$ & $5(2-9)$ & $<0.001$ \\
Total charges & $\$ 25,644(\$ 13,835-\$ 49,423)$ & $\$ 28,310(\$ 14,533-\$ 58,699)$ & $<0.001$ \\
\hline & & Disposition & $<0.001$ \\
Discharge to home & $70.4 \%$ & $68.3 \%$ & NS \\
Discharge to acute care setting & $2.7 \%$ & $2.9 \%$ & NS \\
Discharge to facility & $21.9 \%$ & $21.5 \%$ & $<0.001$ \\
In-hospital mortality/death & $4.0 \%$ & $6.0 \%$ & \\
\hline
\end{tabular}

differences between the patients in the ESRD population itself should be elicited to determine where interventions may help decrease risk factors correlated with pathology.

Vascular calcification is a well-documented phenomenon in ESRD patients which directly contributes to increased cardiac mortality. While the exact mechanism remains unclear, it has been ascribed to changes in vascular smooth muscle correlated with intimal proliferation and atherosclerosis. Warfarin therapy further provokes an unbalancing of calcification factors by decreasing the amount of active vitamin $\mathrm{K}$ in circulation. Vitamin $\mathrm{K}$, aside from its role in the clotting cascade, interacts with Matrix G1A protein to inhibit vascular calcification. A reduction in Matrix G1A protein in rat models leads to substantial vascular calcification $[9,10]$. Furthermore, genetic variation among patients and varying gamma-carboxylation at the glutamate residues result in different phenotypes and associated susceptibility to vascular calcification [9-11].

Our national study aimed to determine the impact of warfarin administration on patients with ESRD. Cardiovascular mortality in particular was of interest given that cardiac events are the primary cause of mortality in patients with ESRD. Our national data shows a significant increase in pathologies rooted in vascular calcification in ESRD patients taking warfarin. Specifically, MI, PVD, CVA, HTN, and valvular disease were all significantly more prevalent in patients taking warfarin. Interestingly, this did not translate to an increase in in-hospital mortality as patients not taking warfarin have significantly higher rates of in-hospital mortality. However, our database manipulation did not allow us to follow up patients in the long term to determine the overall impact warfarin has on mortality in ESRD patients. ESRD patients on warfarin had shorter LOS and were more likely to be discharged to home than to a care facility. This data suggests that warfarin administration may be correlated with an increased risk of developing vascular pathologies though this does not appear to impact all-cause mortality rates.

\section{Limitations}

There are limitations inherent to all administrative databases such as the HCUP-NIS. There can be coding errors, leading to missed diagnoses and procedures, as well as a lack of coding, leading to missing data. The HCUP-NIS is a wellvalidated and rigorously maintained database, and its error rate has been low [7]. This study focused on reporting the differences in comorbid conditions and mortality in ESRD patients on warfarin and those not on warfarin. As the NIS database only documents single admissions, it was not possible to follow up patients in the long term to determine mortality. Furthermore, it is not possible to determine the reason patients were placed on warfarin.

\section{Conclusion}

Patients with ESRD taking warfarin are more likely to have MI, HTN, PVD, valvular disease, DM, CVA, and CHF. They have a decreased LOS, hospital charges, and in-hospital mortality compared to their ESRD counterparts not taking warfarin. Further randomized control trials need to be conducted to determine the impact of warfarin administration on patients with ESRD.

\section{Conflict of Interests}

The authors declare that there is no conflict of interests regarding the publication of this paper.

\section{References}

[1] S. Jono, A. Shioi, Y. Ikari, and Y. Nishizawa, "Vascular calcification in chronic kidney disease," Journal of Bone and Mineral Metabolism, vol. 24, no. 2, pp. 176-181, 2006.

[2] S. M. Moe and N. X. Chen, "Pathophysiology of vascular calcification in chronic kidney disease," Circulation Research, vol. 95 , no. 6 , pp. 560-567, 2004.

[3] M. K. Shea and R. M. Holden, "Vitamin K status and vascular calcification: evidence from observational and clinical studies," Advances in Nutrition, vol. 3, no. 2, pp. 158-165, 2012.

[4] T. Krueger, R. Westenfeld, M. Ketteler, L. J. Schurgers, and J. Floege, "Vitamin K deficiency in CKD patients: a modifiable risk factor for vascular calcification?" Kidney International, vol. 76, no. 1, pp. 18-22, 2009.

[5] T. Krüger, R. Westenfeld, L. J. Schurgers, and V. M. Brandenburg, "Coagulation meets calcification: the vitamin K system," International Journal of Artificial Organs, vol. 32, no. 2, pp. 67$74,2009$.

[6] E. Theuwissen, E. Smit, and C. Vermeer, "The role of vitamin K in soft-tissue calcification," Advances in Nutrition, vol. 3, no. 2, pp. 166-173, 2012. 
[7] Agency for Healthcare Research and Quality, Hospital inpatient statistics, http://hcupnet.ahrq.gov/.

[8] Kidney Disease Statistics for the United Stated, 2014, http:// kidney.niddk.nih.gov/kudiseases/pubs/kustats.

[9] C. Saifan, M. Saad, E. El-Charabaty, and S. El-Sayegh, "Warfarin-induced calciphylaxis: a case report and review of literature," International Journal of General Medicine, vol. 6, pp. 665669, 2013.

[10] D. L. Zimmerman and J. P. Harmon, "Anticoagulant and antiplatelet therapy in patients with chronic kidney disease: risks versus benefits review," Current Opinion in Nephrology and Hypertension, vol. 22, no. 6, pp. 624-628, 2013.

[11] J. Liu, K. L. Ma, M. Gao et al., "Correction: inflammation disrupts the LDL receptor pathway and accelerates the progression of vascular calcification in ESRD patients," PLoS ONE, vol. 8, no. 8, 2013. 


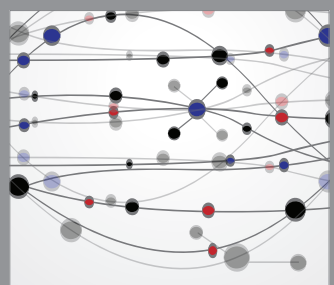

The Scientific World Journal
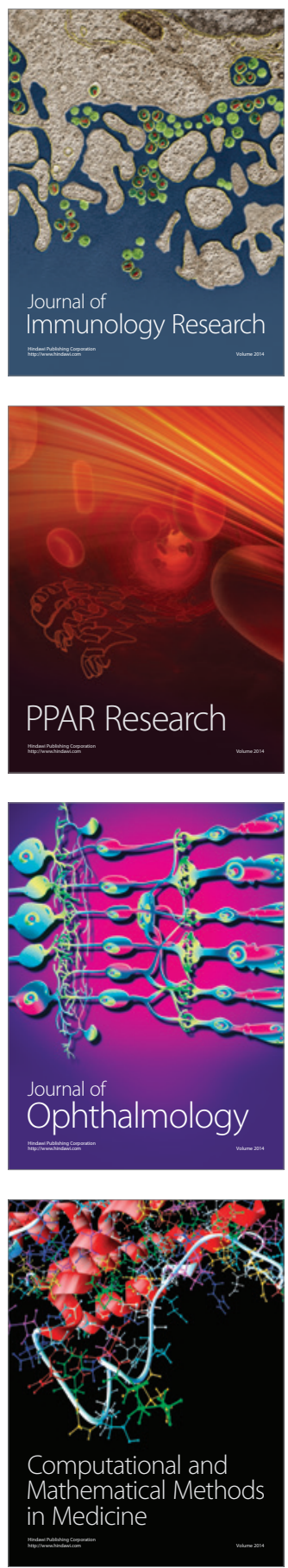

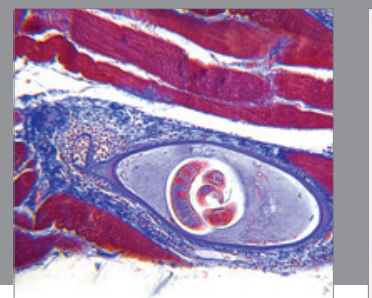

Gastroenterology

Research and Practice
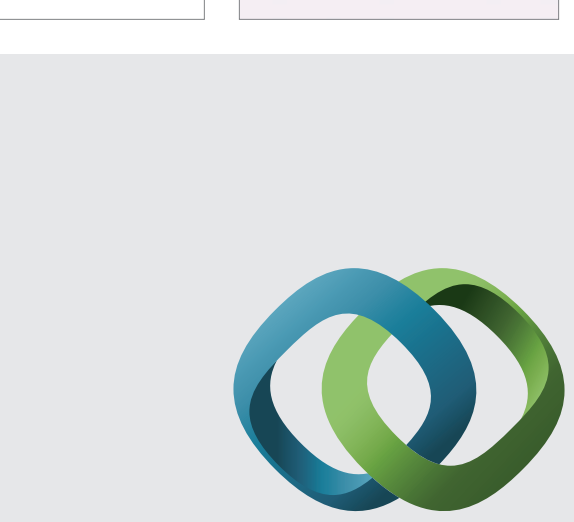

\section{Hindawi}

Submit your manuscripts at

http://www.hindawi.com
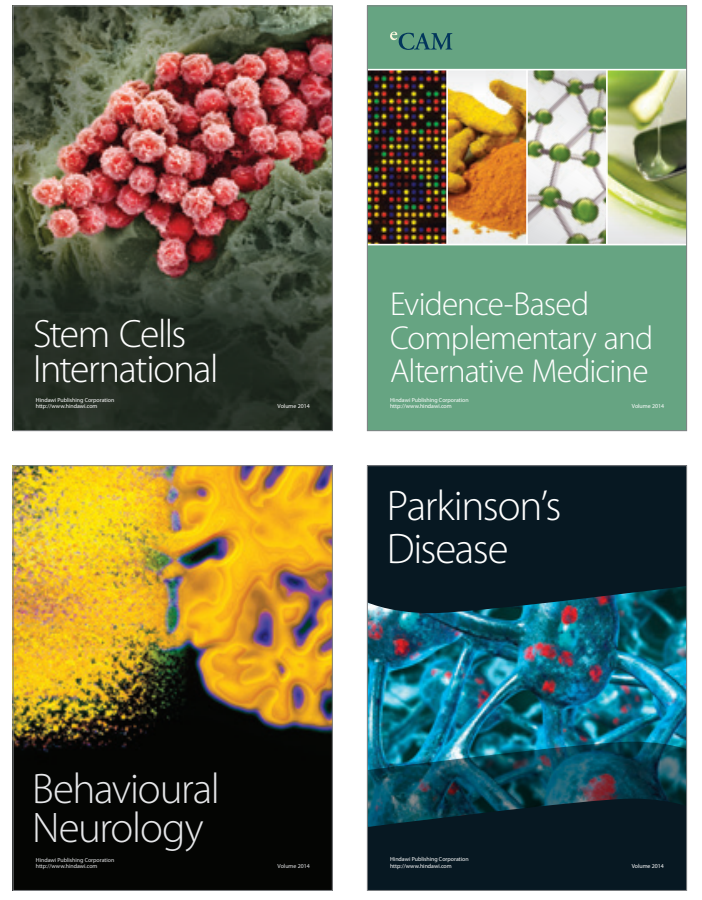
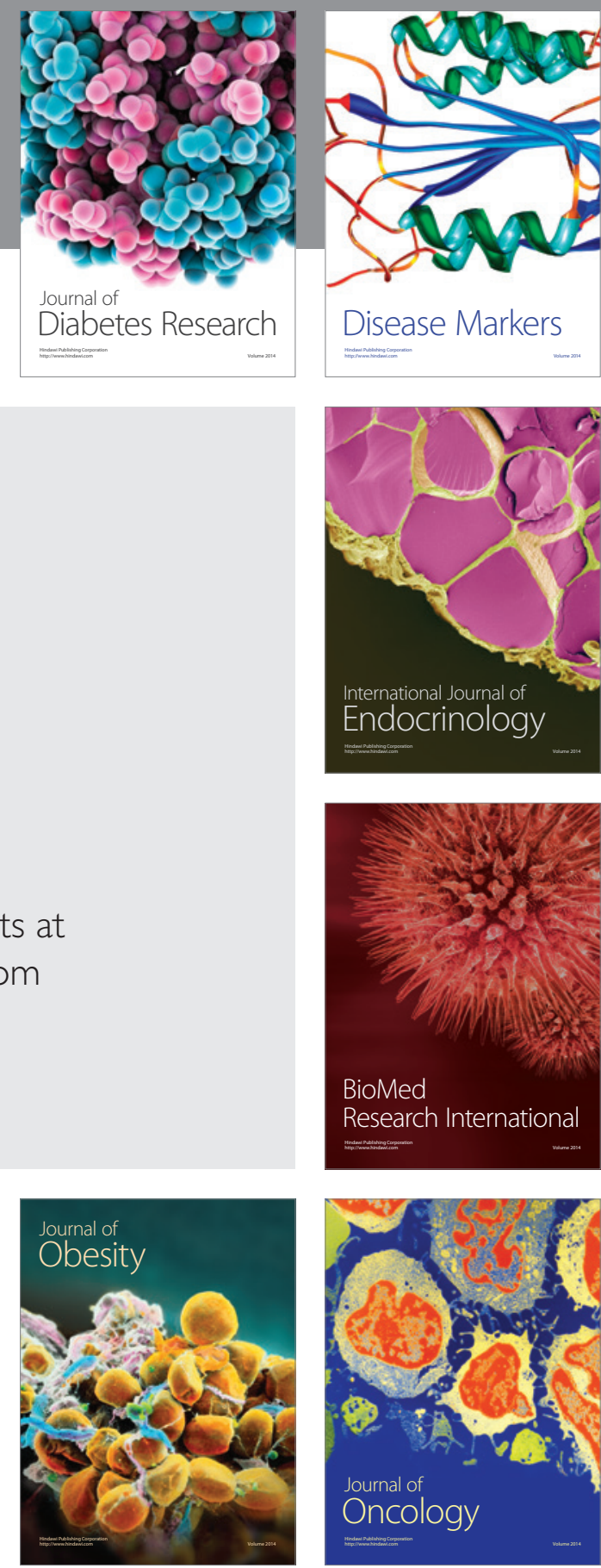

Disease Markers
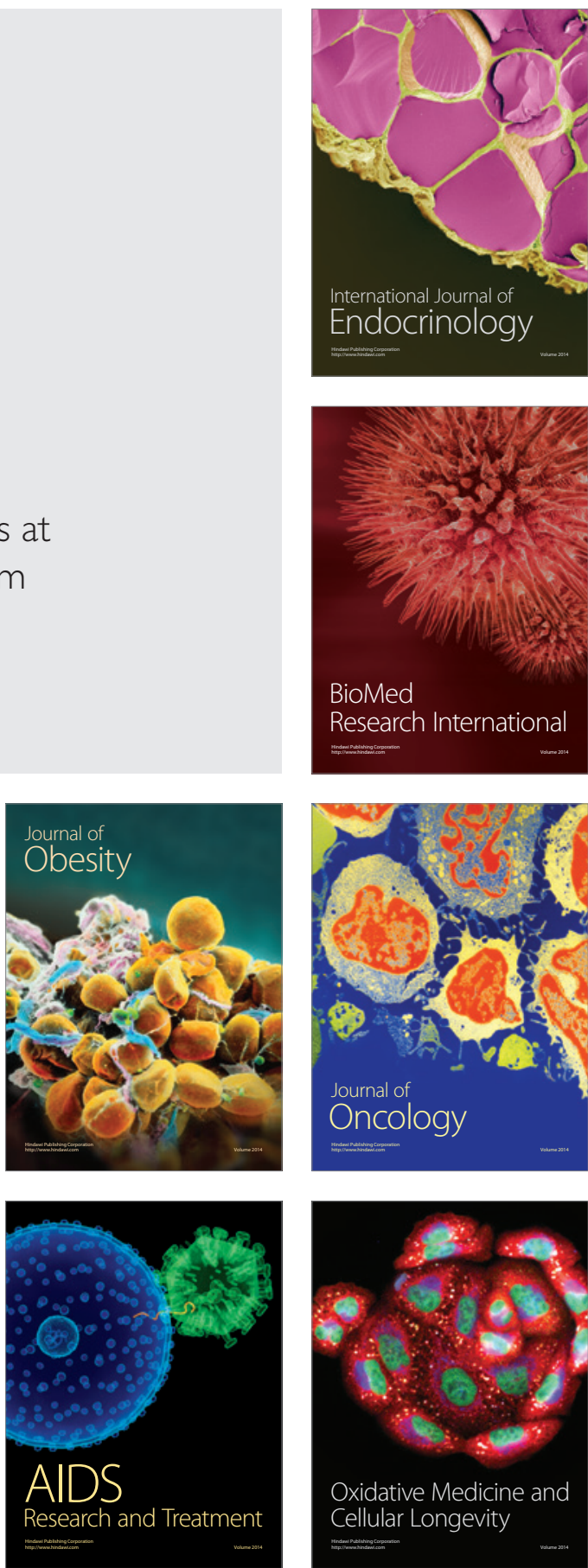